\title{
Come fare lezione su gli aspetti ancora vivi e sensibili della storia passata? La rete delle prospettive
}

\section{di Bjorn Wansink, $\underline{\text { Albert Logtenberg, Geerte Savenije, Elise Storck and Asker Pelgrom }}$}

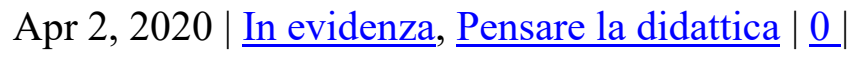

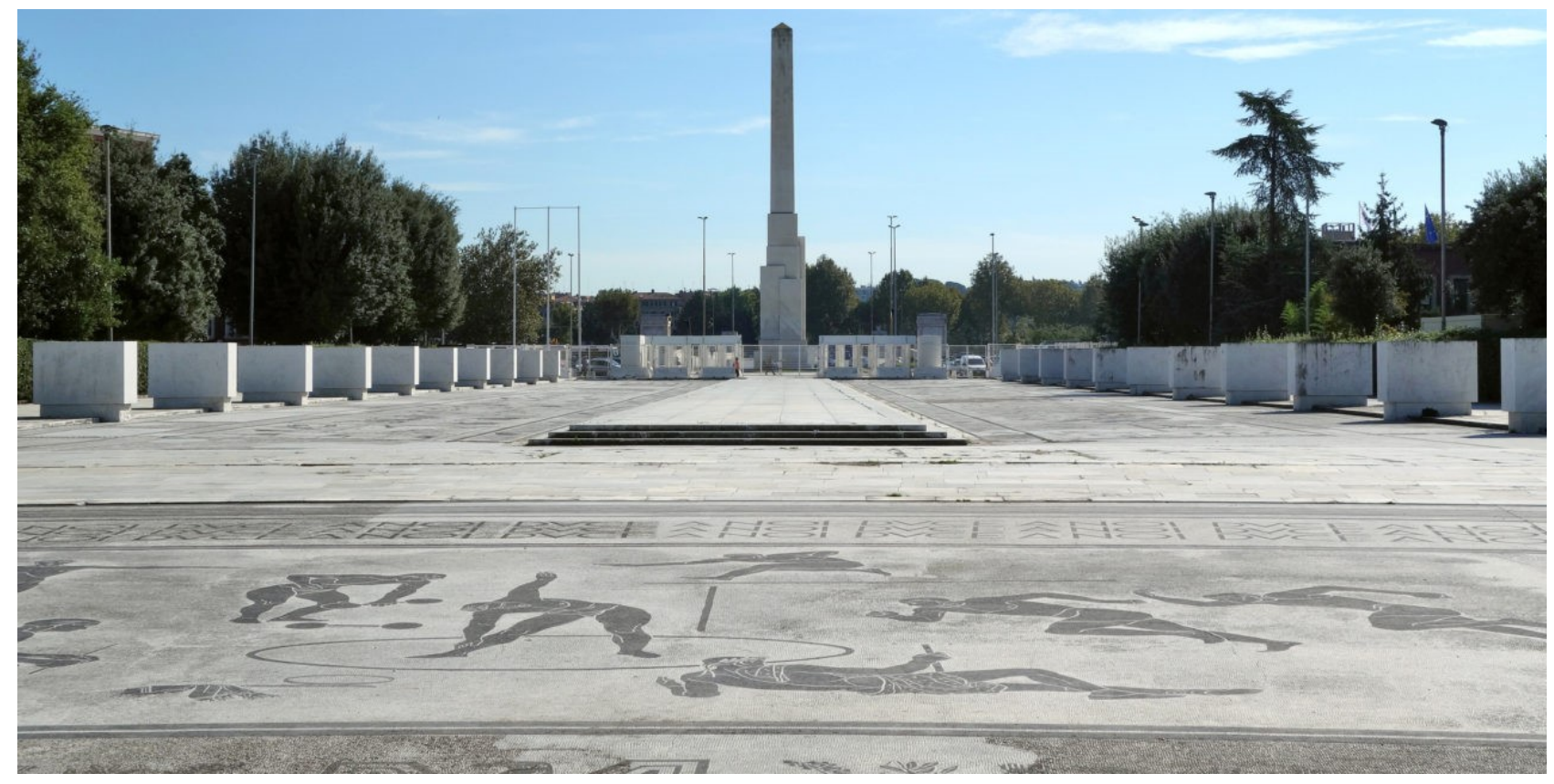

Il Foro italico.

Foto di $\underline{0000 \mathrm{ff}}$ - proprio lavoro, CC BY-SA 3.0, Link

\section{L'articolo è stato tradotto per Novecento.org da Paolo Ceccoli}

$\underline{\text { Scarica la versione originale in inglese }}$

\begin{abstract}
La didattica della storia, soprattutto all'estero, costruisce modelli che possono aiutare i docenti ad affrontare i problemi quotidiani posti dal lavoro pratico nelle classi. Gli autori di questo articolo presentano qui la struttura e le ragioni che li hanno portati a elaborare uno schema di lettura didattica delle questioni storiche ancora sensibili, quelle, per intenderci, della contemporaneità più vicina a noi, o quelle che presentano aspetti etico-morali capaci di rendere le lezioni particolarmente cariche di emotività. La rete delle prospettive, questo il nome del modello, parte dalla constatazione che ci siano sempre argomenti storici che sono particolarmente difficili da affrontare per la loro delicatezza o perché possono riaprire ferite appena cicatrizzate. Molti docenti, proprio per queste difficoltà, non se la sentono di svilupparli pienamente o tendono a sminuirne il valore educativo. Con questo modello gli autori vengono incontro alle esigenze di chi invece vuole farlo mostrando una serie di approcci metodologici possibili.
\end{abstract}


In Italia e nei Paesi Bassi i nervi ancora scoperti delle rispettive storie nazionali hanno recentemente riacceso il dibattito pubblico. Entrambi i paesi hanno assistito a vivaci discussioni sulle commemorazioni e sul ruolo dei monumenti, sugli eroici ammiragli e capitani olandesi di lungo corso o sui personaggi iconici del Risorgimento. La progettazione di musei dedicati a questioni delicate come lo schiavismo nei Paesi Bassi o il fascismo in Italia ha aperto nuove querelles, mentre il passato coloniale dei due paesi dovrebbe motivare a un insegnamento multi-prospettico di questo aspetto della loro storia, sempre più necessario a rendere ragione delle società multiculturali nelle quali viviamo. Gli insegnanti lavorano all'interno di questo contesto: i dibattiti politici e di opinione che sui mezzi di comunicazione di massa promuovono la discussione sull'immigrazione o sull'Islam sollecitano gli educatori a una riflessione sulle proprie scelte didattiche; temi come la storia dell'emigrazione, dell'Olocausto o del conflitto israelo-palestinese sono in questo senso particolarmente sensibili. I docenti, inoltre, si confrontano inevitabilmente nelle loro classi con questioni sollevate dai conflitti sociali, nell'insegnamento dei quali ci si attende un contributo alla formazione di competenze di cittadinanza democratica (Council of Europe, 2018) come la tolleranza, il pensiero critico, la capacità di assumere una prospettiva diversa dalla propria. Tuttavia, proprio quando i docenti sono di fronte alla necessità di toccare questioni sensibili, tali competenze si trovano esposte a pressioni nei confronti delle quali talvolta essi stessi non sanno come reagire. Ne consegue la constatazione di un bisogno: per insegnare le questioni storiche controverse i docenti hanno la necessità di ampliare le loro competenze didattiche.

Nel febbraio del 2019 a Roma Asker Pelgrom (Reale Istituto Neerlandese di Roma) e Bjorn Wansink (Università di Utrecht, Paesi Bassi) hanno organizzato un convegno intitolato The sensitive past, history \& heritage education in Italy and the Netherlands (L'educazione al patrimonio e la didattica della storia e del passato ancora oggi vivo e sensibile in Italia e nei Paesi Bassi). Il convegno si è proposto di indagare come gli insegnanti, i formatori e i ricercatori italiani e olandesi trattino il passato ancora vivo nella didattica della storia o nella didattica museale e del patrimonio. In questo articolo esamineremo una parte dei materiali, delle proposte educative e delle intuizioni di ricerca che sono state presentate al convegno. Presenteremo, per cominciare, una panoramica concettuale di cosa si possa considerare come sensitive, e cioè storia ancora viva e sensibile[1]. In secondo luogo faremo la proposta di una "rete di prospettive" che può essere usata come uno strumento pratico per la progettazione di lezioni su argomenti storici sensibili. Lo strumento è stato pensato sulla base delle finalità e delle sfide che nella pratica odierna i docenti dei Paesi Bassi si trovano di fronte e si fonda su intuizioni tratte dalla ricerca e dall'esperienza della scuola secondaria olandese, nonché dalla formazione degli insegnanti. Riporteremo, infine i risultati della visita- laboratorio che i partecipanti al convegno hanno svolto al Foro Italico a Roma.

\section{CONCETTUALIZZARE E DISCUTERE IL PASSATO VIVO E SENSIBILE}

Quando nella didattica della storia si parla di argomenti sensibili è importante capire che la natura sensibile del passato è sempre legata al contesto. Ciò che è considerato sensibile cambia nel tempo e nello spazio e differisce secondo chi se ne occupa. Per esempio argomenti che non sono ritenuti controversi nel dibattito accademico o tra la maggioranza del pubblico possono diventare controversi o sensibili all'interno di una classe con alunni migranti provenienti da differenti background culturali. Oppure, a causa di differenze regionali, quello che può non essere considerato controverso nei Paesi Bassi può esserlo in Italia.

Quando ci riferiamo agli argomenti sensibili di un passato ancora vivo, distinguiamo tre caratteristiche, che spiegheremo attraverso l'esempio della schiavitù (Sheppard, 2010): 
- L'argomento si riferisce a eventi traumatici e include la messa a fuoco della sofferenza, dell'oppressione e della violenza subita da un gruppo umano. Questo è chiaro nel caso della schiavitù, sia riguardo agli eventi storici che al sistema schiavistico in generale. Senza contare che fenomeni di disuguaglianza e di discriminazione nella società olandese di oggi sono da mettere in relazione alla schiavitù del passato e quindi, quando sono discussi nelle classi, vengono considerati parte dell'argomento in oggetto.

- Si registra qualche forma di identificazione fra coloro che studiano la storia e coloro che vi sono rappresentati. Tale identificazione, fondata sulle identità sociali percepite (Tajfel, 1982), conduce spesso alla negazione di particolari prospettive e alla sottolineatura di altre. Per esempio chi si ritiene olandese potrebbe voler passare sotto silenzio le azioni delle compagnie olandesi trafficanti di schiavi e dei proprietari olandesi di piantagioni e ciò perché tali azioni possono configurarsi come minaccia nei confronti dell'identità sociale e dell'immagine morale positiva del loro gruppo (Bar-Tal, 2017).

- L'argomento produce una qualche reazione morale nel presente. Per esempio quando si insegna la schiavitù da una prospettiva attuale, gli studenti potrebbero sentire il necessario ratificare la necessità di agire per evitare che simili eventi accadano di nuovo o perfino proporsi di porre rimedio agli errori del passato (Savenije, Van Boxtel \& Grover, 2014; Leone \& Sarrica, 2017).

Gli argomenti potenzialmente sensibili come la schiavitù sono spesso in vari modi sottaciuti: dai governi o nei curricoli, dai gruppi sociali o dai singoli insegnanti attraverso l'autocensura (Savenije \& Goldberg, 2019). Sul tema della schiavitù, ad esempio, un insegnante potrebbe negare l'esistenza di prospettive contrarie fra loro per paura di generare discussioni troppo accese o per il desiderio di proteggere una credenza o un valore (Brauch, Leone \& Sarrica 2019). Questi silenzi e questi dinieghi, sociali o politici, sono sempre in qualche modo il prodotto della scelta consapevole di ignorare (Cohen, 2001; Connerton, 2008; Winter, 2010). È perciò importante che gli insegnanti di storia diventino consapevoli della censura sociale e dell'autocensura concernente gli argomenti "spinosi" e si impegnino invece a darne conto dando voce anche agli studenti (Savenije \& Goldberg, 2019).

Le discussioni in classe su argomenti sensibili sono, del resto, una componente essenziale dell'educazione alla cittadinanza democratica poiché incoraggiano gli studenti a partecipare attivamente e criticamente (Oulton et al., 2004). Gli argomenti ancora vivi forniscono un'opportunità di apprendimento e possono contribuire allo sviluppo di abilità quali la capacità di formarsi un'opinione fondata su prove e la capacità di riconoscere, così come di interrogare criticamente, prospettive contrastanti (Goldberg \& Savenije, 2018; Wansink et al., 2019). Inoltre gli studenti stessi pensano che queste discussioni siano importanti e spesso manifestano atteggiamenti positivi nei loro confronti (Hess \& Posselt, 2002).

E tuttavia alcune ricerche negli anni passati hanno dimostrato come gli insegnanti di storia siano spesso poco inclini a sviluppare argomenti percepiti come sensibili e controversi. Questo perché implicano sfide emotive e intellettuali considerevoli (Goldberg, 2017; Savenije \& Goldberg, 2019). Gli insegnanti possono essere spaventati dall'ardore con cui alcuni studenti potrebbero porre in contrasto i loro punti di vista. Da una parte questa diversità di prospettive può essere un'opportunità per un approccio didattico esplicitamente multi-prospettico (Goldberg \& Savenije, 2018), dall'altra le discussioni potrebbero facilmente degenerare, dal momento che le prospettive da cui si guarda il passato sono spesso fortemente correlate con la propria identità e i propri valori (e.g., Epstein, 1998; Peck, 2010). In questo modo, nei casi in cui le classi vivono situazioni di particolare tensione, gli studenti potrebbero trovare molte difficoltà a riflettere criticamente sulla loro stessa prospettiva e essere ancor meno aperti a comprendere visioni differenti e contrastanti (Barton \& McCully, 2012; King, 2009). 
Sembrano esserci due fattori cruciali nell'approccio dei docenti verso argomenti la cui memoria è ancora oggi viva. Il primo riguarda la relazione morale del docente con l'argomento in questione, il secondo la sua esperienza di insegnamento. Alcune ricerche hanno mostrato che la relazione fra le convinzioni degli insegnanti e il loro atteggiamento verso un insegnamento multi-prospettico dipende dall' argomento ed è influenzata da come viene percepito, come un argomento cioè più o meno "delicato" e perciò potenzialmente sensibile da insegnare (Wansink, Ackerman \& Wubbels, 2016). Argomenti percepiti come storicamente "freddi" sono ritenuti più facili da affrontare partendo da diverse prospettive rispetto a quelli più "caldi". E del resto gli insegnanti, se vogliono davvero rendere possibile una discussione multi-prospettica su qualche argomento storico, hanno bisogno di competenze pedagogiche e di conoscenze specifiche affinché la discussione stessa sia fondata sulla valutazione delle fonti e sulla contestualizzazione; se vogliono, in altre parole, fondare l'argomentazione sull'evidenza storica (Goldberg and Savenije 2018; Wansink et al., 2018).

L'esigenza di insegnare agli studenti il ragionamento storico (Van Drie \& Van Boxtel, 2008) è particolarmente impegnativa per gli insegnanti della scuola secondaria al punto che potrebbe persino scoraggiarli dal tentare. In questo senso Kitson e McCully (2005) parlano degli insegnanti capaci di "prendersi il rischio", quelli che accettano la sfida e si pongono come attivatori del pensiero critico attraverso la scelta di impegnarsi nello sviluppo di argomenti storici controversi allo scopo di stimolare la consapevolezza critica degli studenti e promuovere il cambiamento sociale.

È importate non dare per scontato che avere a che fare con argomenti storici sensibili, ancor oggi vivi nel discorso pubblico, pone gravi problemi agli insegnanti di storia. In questo articolo forniremo uno strumento che può aiutare i docenti a prendersi i rischi necessari per affrontare in classe argomenti controversi stimolando il ragionamento storico.

\section{LO STRUMENTO DI PROGETTAZIONE: LA RETE DELLE PROSPETTIVE}

La rete delle prospettive è uno strumento sviluppato da Logtenberg, Storck e Wansink (2018) che può aiutare a concentrare l'attenzione sia sul ragionamento storico sia sulla pertinenza personale per gli studenti, entrambi aspetti di uguale rilevanza nell'insegnamento di argomenti "sensibili". Lo schema concettuale della rete potrebbe ispirare gli insegnanti a perseguire un approccio sistematico verso gli argomenti ancora vivi allo scopo di promuovere il senso della comprensione di diverse narrazioni e/o del cambiamento delle narrazioni del corso del tempo. Esso aiuta a fare in modo che, per ciascun argomento, sia affrontata almeno una prospettiva di ragionamento storico o un aspetto di importanza personale. La rete delle prospettive (vedi figura 1) è composta di tre parti principali:

- i livelli temporali, rappresentati dai cerchi,

- l'imbuto tra astratto e concreto, spiegato nel cerchio grigio interno,

- le otto direzioni di pensiero, dalla A alla H.

Vediamo brevemente di cosa si tratta. 


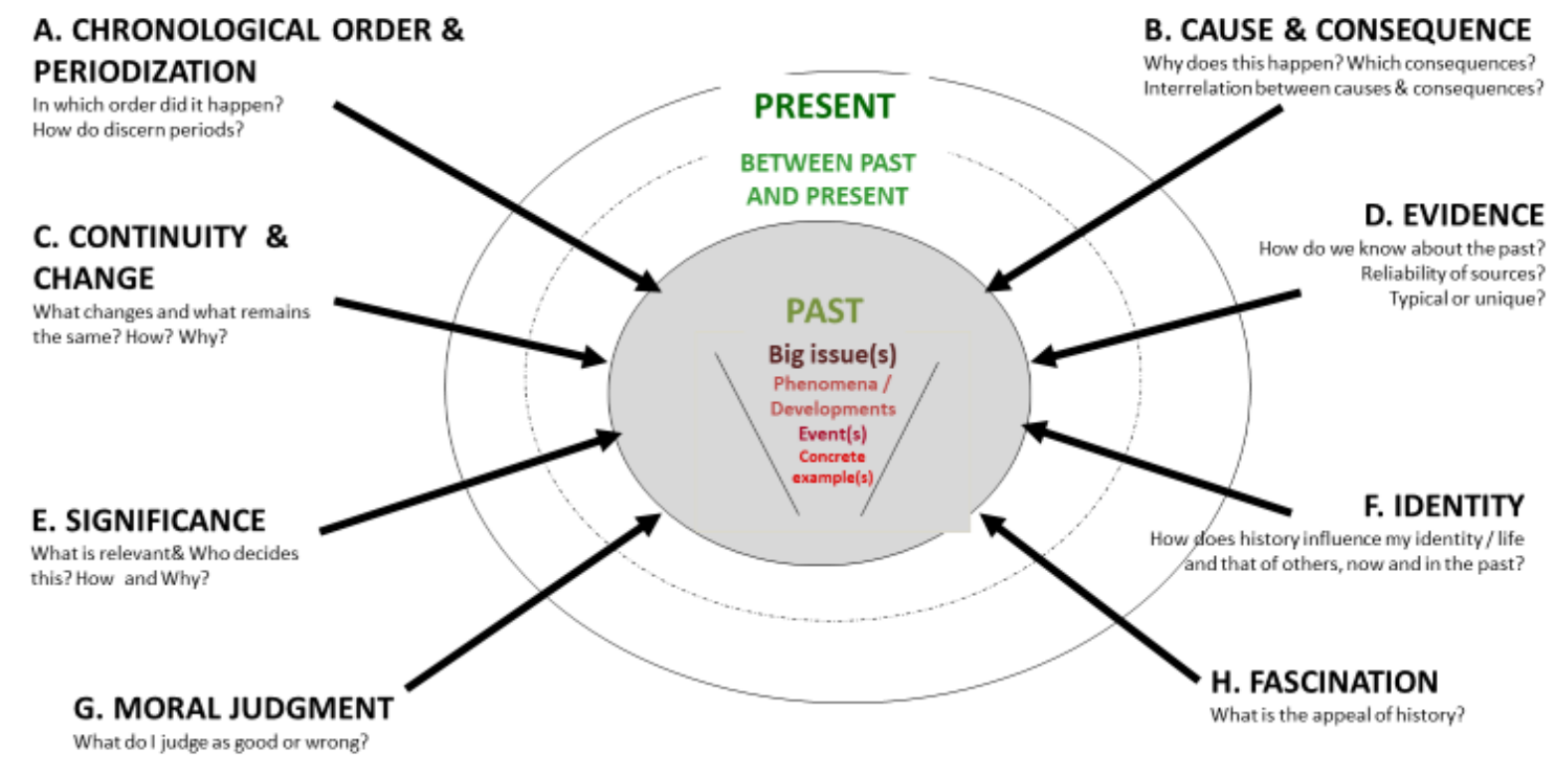

\section{Tre livelli temporali}

I tre cerchi indicano le differenze fra il presente e il passato. Il passato si riferisce a un tema storico in gioco (evento, oggetto o persona) e alle prospettive contemporanee sull'argomento.

Proviamo a fare un esempio: come reagirono i Romani del tempo all'assassinio di Cesare? Il cerchio tra passato e presente si riferisce a un livello temporale non contemporaneo, che ha anche una connessione con la morte di Cesare (l'oggetto storico) e la sua interpretazione (ad esempio quello che ne scrive Edward Gibbon nel 1776). La principale funzione di questo cerchio consiste nell'esplicitare agli studenti il fatto che le narrazioni storiografiche degli eventi storici possono variare nel tempo a seconda della posizione temporale, socio-culturale e politica della persona che costruisce il racconto. L'ultimo cerchio rappresenta il presente dell'oggi nel quale tutti viviamo, studenti, insegnanti di storia, politici e storici compresi. Lo scopo principale di questo cerchio temporale consiste nel far riflettere e rendere criticamente consapevoli gli studenti del fatto che essi non sono solo consumatori di storia, ma contribuiscono anche in proprio alla costruzione del loro passato (Wansink, Ackerman, Zuiker \& Wubbels, 2018).

\section{L'imbuto fra astratto e concreto}

Gli argomenti storici più importanti e le questioni esistenziali più significative sono astratte. Tanto più giovani sono gli studenti quanto più hanno bisogno di esempi concreti tratti da situazioni storiche particolari. Un compito importante per il docente di storia consiste nel compiere scelte esplicite tra i contenuti e gli approcci didattici. L" "imbuto" nel centro della rete può essere usato come uno strumento per istituire connessioni fra esempi concreti del passato e concatenazioni di temi storici più ampi. Per esempio l'assassinio di Cesare può essere usato come un'introduzione concreta a un tema più generale come l'Impero romano, il governo e le questioni esistenziali passate e presenti che riguardano l'esercizio del potere. È importante, quale diretta conseguenza, che l'insegnante definisca il contesto storico spazio-temporale degli argomenti e degli sviluppi storici più importanti, così come dei temi più propriamente sociali: ciò permette agli studenti di indagare il contesto storico con precisione crescente. Domande mirate su argomenti e protagonisti della storia forniscono indicazioni che inducono a riflettere su sviluppi e fenomeni più astratti. L'imbuto 
visualizza la nozione che i temi e le questioni più importanti possono essere compresi meglio se si conoscono esempi tratti dai contesti storici concreti e che, d'altra parte, i processi, i reperti, i protagonisti e gli eventi concreti della storia possono essere compresi meglio quando vengono collocati nel quadro di tematiche più vaste (Logtenberk, Storck \& Wansink, 2018).

\section{Gli spicchi della rete}

Gli "spicchi" nella rete delle prospettive visualizzano le prospettive che funzionano come direzioni del pensiero, quando intendiamo studiare il passato. La rete include otto concetti basati sul lavoro di Seixas e colleghi (2013) e su idee di chi scrive (ma chi scrive...è il traduttore o il tradotto?):
A. cronologia
B. causa e conseguenza
C. continuità e cambiamento
D. prova
E. rilevanza
F. identità
G. giudizio morale
H. fascino

Proviamo a definire brevemente ciascuno di questi elementi.

A. Tempo e cronologia. Questa prospettiva riguarda la comprensione del tempo storico e della sua sequenzialità espressa in epoche Senza questo concetto è impossibile categorizzare il tempo suddividendolo in epoche utilizzabili come cornici di riferimento. Il tempo storico è organizzato in narrazioni che hanno un inizio e una fine ed è perciò una dimensione differente del tempo rispetto al tempo oggettivato dall'orologio o al tempo soggettivo (quanto tempo mi sembra sia passato). Importanti sviluppi sistematici di questa prospettiva sono la cronologia, la periodizzazione e l'anacronismo (Wilschut, 2011; De GrootReuvekamp et al., 2014).

Le domande che si possono associare a questa prospettiva sono: quanto è durata quest'epoca? In che ordine si susseguono le epoche?

B. Causa e conseguenza. Questa prospettiva riguarda il ragionamento causale. Per questo approccio è importante riconoscere che cause e conseguenze sono sempre complesse e interconnesse. Possono variare per forza e intensità così come possono indebolirsi nelle loro relazioni reciproche; le conseguenze, inoltre, possono diventare a loro volta cause in un secondo momento (Stoel, Van Drie \& Van Boxtel, 2017). Questa è anche la prospettiva dalla quale può essere indagato il ruolo delle circostanze culturali, politiche e di governo, economiche e sociali.

Le domande che si possono associare a tale approccio sono: quali sono le conseguenze a breve e lungo termine di un evento? Quali furono le diverse cause e cosa sarebbe successo se qualcuna di queste non fosse avvenuta? Quali furono le cause primarie e quali le secondarie? Quali furono le intenzioni specifiche degli attori del passato?

C. Mutamento e continuità. Questo approccio concerne le interconnessioni fra gli eventi che permangono e quelle che si modificano nel corso del tempo. Potrebbe riguardare inoltre l'intuizione della velocità e dei ritmi della storia. Ad esempio in alcuni processi rivoluzionari i cambiamenti intervenuti sono evidenti, mentre in altri casi è più difficile discernere cosa è cambiato e cosa no (Counsell, 2011). Ciò che qualcuno percepisce come un cambiamento, qualcun altro può sentirlo come una continuità. $\mathrm{O}$, se consideriamo l'apprezzamento, quello che per qualcuno è progresso per qualcun altro è esperienza di declino. Inoltre è importante che gli studenti comprendano quanto i cambiamenti nel corso 
del tempo siano costituiti da processi, cioè che alcuni cambiamenti non avvengono improvvisamente.

Le domande che si possono associare a questo approccio sono: perché qualcosa è cambiato e qualcos'altro no? Quali momenti di svolta possono essere distinti in un processo di sviluppo?

D. La prova. Le storie pretendono di essere ricostruzioni del passato basate sulle fonti. Questo approccio riguarda l'uso delle fonti e delle prove e considera attentamente l'uso dell'informazione nelle fonti e sulle fonti: come è stata costruita la storia? Gli studenti dovrebbero essere capaci di dimostrare le loro intuizioni circa le ricostruzioni del passato attraverso la decostruzione delle narrazioni disponibili, usando materiale documentario differente e/o nuovo o facendo domande differenti e/o nuove alle fonti utilizzate per quelle ricostruzioni. È meno comune, ma non del tutto inusitato, che agli studenti sia richiesto di ricostruire in autonomia il passato. Ciò può accadere, per esempio, quando ci si aspetta che svolgano la loro ricerca per un compito pratico o per il loro lavoro di gruppo. La storia e le intenzioni dell'autore, insieme al contesto della fonte, devono essere esaminati con la massima attenzione quando si usano fonti differenti (Wineburg, 2001).

Le domande che si possono associare a questo approccio sono: cosa significa questa informazione? Cosa manca o cosa è stato deliberatamente omesso? Chi fornisce questa informazione e con quale intento? Per quali credenze, gruppi di persone o situazioni questa informazione è rappresentativa? L'informazione in questione si accorda con ciò che hanno da dire altre fonti in proposito? Quale interpretazione sembra più valida e quale prova la sostiene?

E. Rilevanza. Questa prospettiva riguarda l'importanza della storia per noi oggi e per gli uomini del passato. Come si determina quale storia sia importante e quale no? Considerando che la rilevanza della storia è caratterizzata da una relazione flessibile con il passato, i suoi criteri cambiano nel corso del tempo. Ciò che poteva essere di grande valore per un certo gruppo nel passato in un'epoca differente potrebbe essere sottovalutato da un altro gruppo. Le domande che si possono associare a questo approccio sono: Perché desideriamo conoscere un certo argomento? Quale racconto del passato è così importante da voler essere conservato nella memoria sociale? Come facciamo a decidere quale aspetto del passato è importante conoscere? La rilevanza può essere una cosa profondamente personale per un individuo, ma può essere anche un costrutto generato nella società da gruppi più ampi.

F. Giudizio morale. È importante chiedersi come il passato possa aiutarci a comprendere e dare forma al presente. Perché impariamo dal passato? Quando lo facciamo è molto difficile giudicare inumani o lodevoli secondo le norme e i valori attuali i comportamenti dei nostri predecessori. Un problema morale potrebbe essere, in questo senso, porsi la questione se $\mathrm{i}$ discendenti degli schiavi abbiano titolo a qualche forma di risarcimento. Dal momento che le norme e i valori sono a loro volta soggetti nel corso del tempo a preconcetti e cambiamenti, può essere utile considerare i giudizi impliciti e espliciti che gli storici formulano sui loro oggetti di studio.

Le domande che si possono associare a questo approccio sono: cosa penso di quello che la gente ha fatto e pensato nel passato? Cosa considero giusto e cosa sbagliato? In che misura questi valori divergono da quelli del passato? In che misura siamo responsabili per gli atti compiuti nel passato e perché?

G. Identità. La storia può aiutare gli studenti a scoprire i propri valori e ideali e a svilupparsi come persone in grado di sostenere la propria posizione nella società. Per ottenere questo risultato la conoscenza del passato dovrebbe essere esplicitamente connessa alla vita degli studenti e alla società della quale fanno parte (Van Straten et al., 2016). Una significativa narrazione storica può essere utilizzata nel contesto di una ricerca personale sulla propria identità e sulla comprensione dello sviluppo sociale. Per quanto riguarda la storia come 
materia scolastica è importante determinare la prospettiva dalla quale la narrazione storica rappresenta $\mathrm{i}$ fatti. Chi sta raccontando la storia, chi è il soggetto di questa storia e a chi si rivolge? Gli insegnanti si rivolgono a studenti con diversi background, che possono avere qualcosa in comune, ma anche una relazione individuale e specifica con il passato (Grever $\&$ Ribbens, 2007). Se l'attenzione a queste diversità viene a mancare è probabile che sia evidenziato solo un punto di vista o che il passato sia discusso in termini dicotomici "noiloro". Ci si deve assicurare che, quando si discute un argomento, si tenga conto sia di chi si trova nelle aule che di chi è oggetto del racconto storico.

Le domande che si possono associare a questo approccio sono: qual è l'influenza del contesto storico sulle azioni, i pensieri e la vita delle persone? In che modo la storia ha determinato la mia vita e il mondo intorno a me?

H. Fascino. Infine il nostro interesse per il passato può essere causato dal fascino che avvertiamo nei confronti di eventi, abitudini e oggetti. Quando si studia la storia può essere utile chiedersi perché qualcosa sembra bello, attraente o offensivo. Eventi, persone e oggetti del passato possono influenzare le vite e i pensieri del presente. L'importanza del passato, dunque, non riguarda solo la sua funzione simbolica, ma anche le relazioni personali che le persone intrattengono con la storia. Sotto questo aspetto gli storici parlano talvolta della "sensazione storica", che fa sembrare possibile un contatto diretto con il passato (Huizinga, 1950). Emozioni quali indignazione, disgusto o ammirazione possono anche essere connesse ai modi con cui sperimentiamo il passato e spiegare perché attrae il nostro interesse. Le domande che si possono associare a questo approccio sono: che cosa trovo di affascinante in questa storia? In che modo questo evento/storia/oggetto mi influenza? Perché lo trovo attraente o offensivo?

\section{Esempio: un laboratorio}

Nel corso della conferenza si è tenuto un laboratorio nel quale partecipanti italiani e olandesi sono stati stimolati a usare la rete allo scopo di discutere diverse prospettive riguardo a un oggetto del patrimonio storico concreto, ancor oggi vivo e sensibile. Il laboratorio è iniziato con la discussione sul caso della mummia sbendata di un bambino vissuto nell'antico Egitto. Questo caso è stato deliberatamente scelto come esempio di argomento sensibile, e tuttavia non così vivo da scatenare accese controversie morali. Potrebbe perciò funzionare come punto di partenza adeguato per introdurre la rete delle prospettive. Gli argomenti eccessivamente sensibili potrebbero infatti ostacolare, attraverso le forti emozioni che suscitano, la discussione sulla multi-prospettività (Wansink et al, 2017).

La mummia di questo bambino dell'antico Egitto appartiene alla collezione del Museo Nazionale Olandese dell'Antichità (RMO) fin dal 1828. Esposta nella collezione permanente, è stato uno dei pezzi più amati dal pubblico; fino al 2016 quando il museo ha deciso di rimuoverla, dopo che un pubblico dibattito aveva sollevato la questione morale se fosse appropriato esibire i resti di un bambino morto. Prevalse l'opinione che oggi non dovrebbe essere più consentito poter mostrare il cadavere di un bambino.

La discussione di questo caso durante il laboratorio (la domanda era: la mummia bambina dovrebbe ancora essere esposta?) ha visto il confronto di molti punti di vista. Molti partecipanti hanno risposto affermativamente mettendo l'accento sulla sensazione e l'importanza storica dell'oggetto (il termine "oggetto" è in se stesso significativo), mentre altri si sono messi nei panni del bambino o dei suoi genitori, argomentando che essi non avrebbero voluto che il corpo del loro figliolo fosse esposto in un museo. 
In casi come questo la discussione produce molte domande che riguardano il ruolo dei musei nell'esporre o non esporre i loro oggetti, l'intenzione originale di chi ha prodotto quel certo oggetto (gli antichi Egizi probabilmente non avrebbero voluto che le loro mummie finissero in un museo), gli effetti che tali oggetti possono produrre su differenti tipi di visitatori, le diverse ragioni per cui un oggetto (dopo anni di esposizione) improvvisamente diventa troppo sensibile per essere mostrato.

Infine questi interrogativi conducono alla domanda più generale: come si trattano i resti umani? La rete dei significati può aiutare a rendere espliciti tali problemi per dare senso alle diverse prospettive di pensiero nel corso del tempo, ma anche per esplicitare prospettive più complesse (l'uso delle fonti e la questione delle prove).

Dopo aver discusso della mummia concentrandosi sul livello temporale del "presente", il conduttore del laboratorio ha mostrato come questo caso possa essere usato per affrontare anche altri livelli temporali.

Un insegnante potrebbe, ad esempio e secondo l'età e il livello degli studenti, scegliere di affrontare il contesto nel quale il ragazzo mummificato è vissuto ed è morto, cioè il livello temporale del passato. Le domande proposte dalla parte sinistra della rete di prospettive possono guidare il processo di apprendimento, la selezione delle fonti e la scelta dei materiali di supporto. Possono riguardare il processo di mummificazione, ma anche il contesto politico, economico e sociale che ha permesso questo tipo di pratiche.

Un'altra opzione di ricerca potrebbe essere studiare il modo nel quale i resti umani sono stati trattati nel corso del tempo (livello temporale tra passato e presente), fornendo una selezione di fonti sulla mummificazione, il nuovo interesse verso l'antico Egitto all'inizio dell'Ottocento (Napoleone, la formazione degli stati nazione, il nazionalismo e la storia dei musei moderni) e il nuovo modo di trattare le mummie. Questo approccio permette agli studenti di riflettere sulla continuità e i cambiamenti avvenuti nel trattare i resti umani, così come l'uso delle caratteristiche definitorie e dei concetti collegati come principi organizzativi (società urbana, pensiero illuminista nel Settecento, formazione delle nazioni e nazionalismo), temi anch'essi obbligatori nel curricolo di storia nella scuola olandese.

\section{Una visita al Foro Italico. Un'opportunità inaspettata?}

Dopo il laboratorio tutti i partecipanti hanno visitato il Foro Italico a Roma. Gli autori di questo articolo non pretendono di essere degli specialisti nel campo della storia fascista e dei suoi lasciti. Tuttavia, come abbiamo fatto nel laboratorio durante la conferenza, vorremmo invitare i lettori di Novecento.org a riflettere sulla praticabilità e l'utilità del nostro modello presentato. Desideriamo perciò provare a utilizzarlo per discutere di un sito come che possiede, come il Foro Italico, vestigia del Ventennio ancora vive e controverse, simili ad altre che si possono trovare in tutta 1'Italia.[2] Fra i molti esempi di arte e di architettura fascista nella zona hanno catturato la nostra attenzione $\mathrm{i}$ mosaici progettati da Angelo Canevari, Achille Capizzano, Giulio Rosso e Gino Severini negli anni tra il 1934 e il 1938 (vedi figura 1). Un passante straniero impreparato come noi è facilmente trascinato a formulare giudizi indignati e senza sfumature sul fatto che questi mosaici sulla storia del fascismo siano ancora esposti (e siano stati persino restaurati nel 2007) e, allo stesso tempo, potrebbe essere affascinato dalla loro bellezza o dalle capacità tecniche di chi li ha realizzati (livello temporale del presente).

Dopo questa prima risposta affettiva e emozionale, l'uso della rete delle prospettive spinge a prendere in considerazione in maniera più dettagliata e sistematica il manufatto e può sollevare 
questioni sul contenuto delle sue raffigurazioni. Nel caso dell'immagine rappresentata nella foto (figura 1), il mosaico si riferisce con ogni probabilità alle intimidazioni e alle violenze fasciste seguite al biennio rosso del 1919-1920 (livello temporale del passato). L'analogia con l'episodio di San Giorgio e il Dragone è interessante perché si riferisce a una cornice culturale differente e meno ovvia, innescando una riflessione attraverso i tempi (livello temporale tra passato e presente).

Tale oggetto, insomma, potrebbe introdurre inedite domande sulla propaganda fascista e sul fatto che non venivano utilizzati esclusivamente motivi e tecniche importate dalla classicità (continuità $\mathrm{e}$ cambiamento), ma anche altri riferimenti culturali. L'opera consente anche di considerare la prospettiva storica allo scopo di comprendere il contesto storico del tempo in cui il mosaico fu realizzato e di porsi una serie di domande su Roma e l'Italia dalla caduta del Fascismo ad oggi e sul loro rapporto con l'eredità lasciata dal regime. La domanda fondamentale sulla quale coinvolgere gli studenti e attivare il ragionamento storico potrebbe essere perciò la seguente: dobbiamo cancellare un monumento del passato o una narrazione specifica perché ci mette a disagio o perché è ancora troppo viva e sensibile?

Possiamo usare in questo modo la rete delle prospettive con i nostri allievi? Gli studenti lavoreranno sulla questione chiave se il passato possa o non possa essere cancellato. Per poter costruire la loro argomentazione storica su questo problema gli allievi potrebbero:

1. studiare le fonti che ricostruiscono le idee dei realizzatori e dei testimoni dei mosaici e determinare in che misura le loro idee fossero rappresentative;

2. descrivere fino a che punto queste idee cambiassero nel corso del tempo;

3. determinare la rilevanza personale (contemporanea) e storica di questi mosaici;

4. formulare una visione più motivata della questione.

Noi crediamo che questo possa essere un compito fecondo per ammorbidire le risposte emotive e giungere a formulare approcci storici più inclusivi. È per questo motivo che invitiamo i lettori di questo articolo a condividere con noi le loro impressioni sull'argomento.[3] 


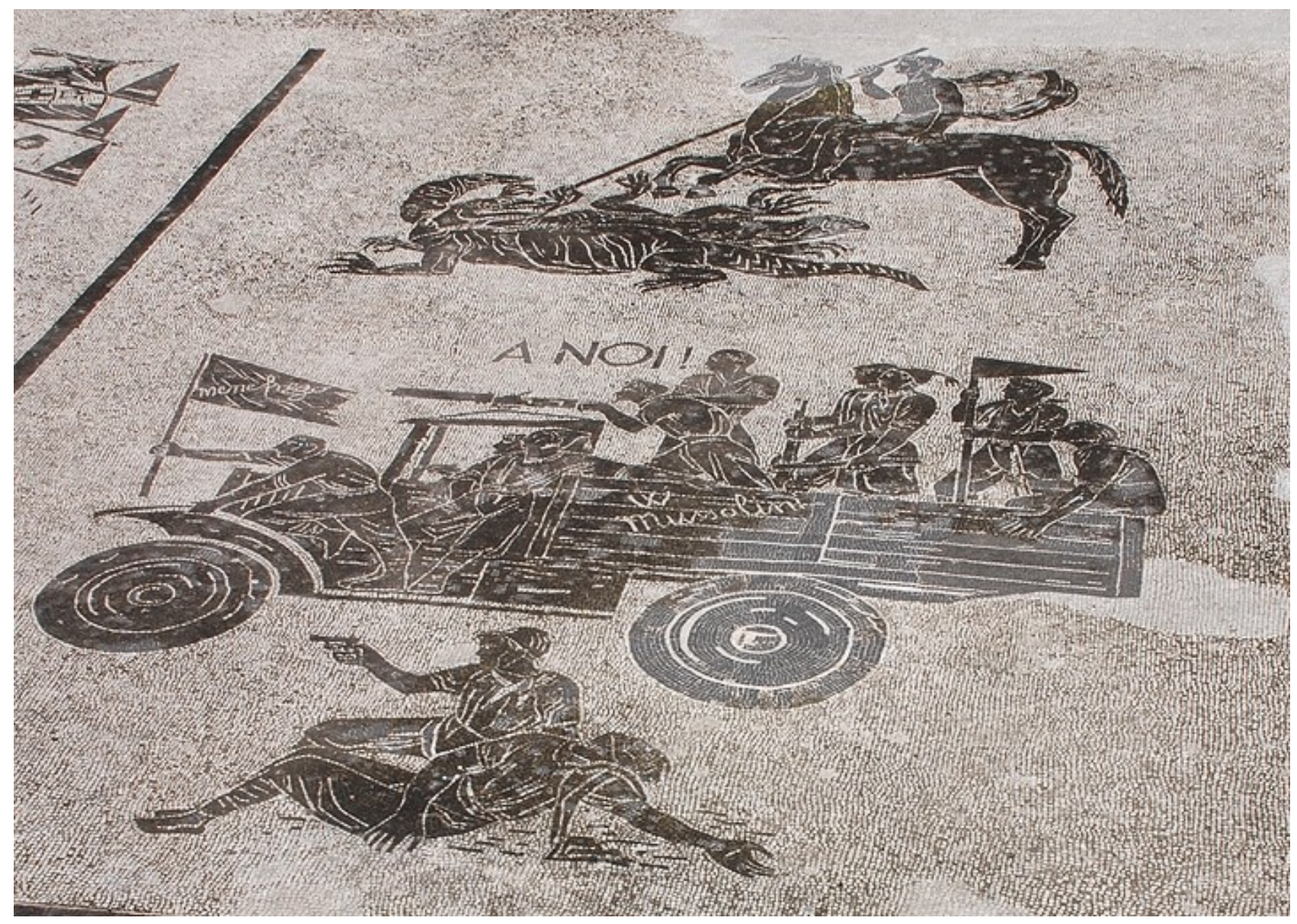

Figure 1: Dettaglio dei mosaici del Foro italico. Foto degli autori.

\section{Conclusione}

Insegnare aspetti controversi della storia può suscitare forti emozioni e generare prospettive in conflitto fra di loro. Le ricerche mostrano che molti insegnanti riferiscono di essere capaci di affrontarli mentre altri docenti fanno più fatica. Abbiamo rilevato che questi insegnanti possono sentirsi insicuri trovando difficile organizzare sul piano didattico le loro lezioni. Per aiutarli gli autori di questo articolo hanno elaborato uno strumento (la rete delle prospettive) fondato su riflessioni teoriche e intuizioni pratiche. Nello studio di argomenti controversi questo strumento consente di ampliare il pensiero storico degli studenti. Le ricerche che chi scrive sta conducendo sull'uso pratico dello strumento e sulle esperienze pratiche dei docenti e degli studenti nei corsi per diventare insegnanti sono incoraggianti. Abbiamo cercato di mostrare come le indicazioni fornite dallo strumento possano davvero aiutare i docenti a compiere scelte consapevoli quando insegnano argomenti particolarmente delicati.

Bisogna tuttavia ammettere che per comprendere come funziona e come si possa applicare correttamente lo strumento occorra tempo. Ci siamo resi conto che gli insegnanti possono essere disorientati dalle varie direzioni che possono prendere quando progettano le loro lezioni con la rete delle prospettive.

Non a caso per questo motivo in Olanda si tengono corsi di formazione sull'argomento. All'inizio si suggerisce agli insegnanti di scegliere una sola direzione e di orientarsi verso l'approccio più semplice possibile. Osservando quale dei tre livelli temporali i docenti privilegiano abbiamo notato che per lo più si indirizzano verso il livello del passato, focalizzandosi sull'oggetto storico posto 
nella sua temporalità, per esempio la mummia nell'Antico Egitto. In alternativa prediligono concentrarsi sul presente chiedendo agli studenti di esprimere le loro opinioni sulle mummie.

Coerentemente con alcune delle ricerche citate i docenti trovano difficile progettare lezioni che si occupino della circolarità del rapporto passato-presente (per esempio come le mummie erano percepite e usate nel XIX secolo). In ogni caso noi pensiamo che il livello temporale del rapporto tra passato e presente, soprattutto quando insegniamo argomenti sensibili vivi ancora oggi, sia importante. Osservando i processi storici dal livello temporale del rapporto tra passato e presente, le prospettive storiografiche che si possono proporre sono in grado rendere più positive e feconde discussioni spesso dominate da posizioni polarizzate dicotomicamente (passato contro presente, sensibile contro scontato).

In relazione alla storia sensibile ancora oggi, proponiamo che i docenti debbano riflettere soprattutto sulle direzioni di pensiero, i giudizi morali, la rilevanza, l'identità e le prove. Innanzitutto perché queste riflessioni innescheranno discussioni accese nelle classi. In secondo luogo perché la stessa relazione dei singoli insegnanti con gli argomenti storici può influenzare la loro strategia di insegnamento e la progettazione delle loro lezioni. Noi pensiamo che usare lo strumento che abbiamo progettato aiuti gli insegnanti a riflettere sulla relazione epistemologica e morale con l'argomento sensibile che devono insegnare.

Infine abbiamo proposto che gli insegnanti, quando iniziano a utilizzare la rete delle prospettive per la progettazione delle loro lezioni, partano da un caso storico che possa essere percepito come ormai chiuso, intendendo con questo termine una narrazione che non produca forte reazioni emotive fra $\mathrm{i}$ docenti o fra gli studenti (come nel caso della mummia bambina). I casi "freddi" possono essere usati al fine di insegnare agli studenti il pensiero storico in un ambiente di apprendimento privo di rischi emotivi. Più tardi queste stesse abilità possono essere applicate in ambienti di apprendimento più stimolanti, nella discussione di argomenti più vivi e sensibili.

Gli autori desiderano ringraziare Paolo Ceccoli per la sua traduzione italiana del loro articolo.

Note:

[1] Traduciamo così l'inglese sensitive poiché la traduzione letterale italiana "sensibile" potrebbe risultare poco chiara.

[2] Si pensi ad esempio alla vicenda di Bolzano e del suo "Monumento Alla vittoria". Vedi: https://it.wikipedia.org/wiki/Monumento alla Vittoria (Bolzano) e https://www.huffingtonpost.it/entry/a-bolzano-monumento-di-epoca-fascista-coperto-con-una-frasedi-hannah-arendt_it_5cc1d564e4b0e68bc67c8580

[3] Si possono interpellare direttamente gli autori ai seguenti indirizzi mail: askerpelgrom@knir.it e/o a B.G.J.Wansink@uu.nl 